\title{
Adnominale Adverbien im europäischen Vergleich
}

\begin{abstract}
Die adnominalen (attributiven) Verwendungsmöglichkeiten von temporalen und lokalen Adverbien im Deutschen werden untersucht und mit denen aus vier anderen europäischen Nachbarsprachen - Englisch, Französisch, Polnisch, Ungarisch verglichen. Gezeigt wird, wie diese Sprachen unterschiedliche Anbindungsstrategien nutzen, um Adverbien in attributiver Funktion einsetzen zu können. Drei solcher Strategien werden unterschieden: Juxtaposition, Adjektivierung und formale Verknüpfung. Die Anbindungsstrategien sind in den Vergleichssprachen unterschiedlich verteilt und in unterschiedlichem Maße dominant. Verfügt eine Sprache über zwei oder mehr Anbindungsstrategien, so können diese in Abhängigkeit von der semantischen Teilklasse des Attributs mit verschiedenen semantischen Beschränkungen und Effekten korreliert sein. Diese bezeichnen wir als temporale bzw. lokale Kompatibilität, Persistenz und Oppositivität. Es lassen sich z.T. übereinzelsprachlich bestimmte Form-Funktions-Korrelationen zwischen Anbindungsstrategien und semantischen Beschränkungen bzw. Effekten feststellen. So können adjektivische und formal verknüpfte Attribute Persistenz und Oppositivität kodieren, juxtaponierte dagegen grundsätzlich nicht.
\end{abstract}

\section{Einleitung ${ }^{1}$}

Adverbien sind Wörter, deren Funktion primär darin besteht, andere Wörter oder Phrasen semantisch zu modifizieren. Diese Eigenschaft teilen sie mit den Adjektiven. Beide Wortarten unterscheiden sich nach traditioneller Auffassung darin, welche Ausdrücke durch sie typischerweise modifiziert werden: Die Domäne der Adjektive sind Nomina, die der Adverbien Verben bzw. Verbalphrasen (einschließlich Sätze) oder andere Adjektive. ${ }^{2}$ In diesem Sinne charakterisieren Ramat/Ricca (1998, S. 187) die Funktion von Adverbien aus typologischer Sicht:

1 Dieser Aufsatz ist im Rahmen des Projekts „Grammatik des Deutschen im europäischen Vergleich“ (Leitung: Gisela Zifonun) am Institut für Deutsche Sprache entstanden. Für Hinweise und Anmerkungen danken wir Peter Gallmann sowie unseren Kollegen Christine Günther, Adriano Murelli, Renate Raffelsiefen, Bernd Wiese und Gisela Zifonun. Besonderer Dank gilt unseren Informanten für die Vergleichssprachen: Séverine Adam, Martine Dalmas, Cécile Delettres (Französisch), George Smith (Englisch), Ewa Drewnowska-Vargáné, Marek Konopka, Robert Mroczynski, Justyna Śliwa (Polnisch), Péter Kappel, Krisztina Molnár (Ungarisch).

2 Gelegentlich werden auch Adverbien zum Anwendungsbereich adverbialer Modifikatoren gerechnet (vgl. Curme 1922, S. 338, Huddleston/Pullum 2002, S. 562, Grevisse 1993, S. 1346). Im Deutschen ist diese ad-adverbiale Funktion (vgl. sehr oft) hauptsächlich auf Intensitätspartikeln beschränkt, für die die ad-adverbale Funktion (vgl. Sie haben sebr geweint.) wiederum sekundär ist (vgl. Zifonun/Hoffmann/Strecker 1997, S. 56). 
Functionally, adverbs are modifiers of predicates, other modifiers or higher syntactic units. In other words they add information to other linguistic elements which can stand on their own, semantically as well as syntactically.

Die Grammatiken der Einzelsprachen weisen jedoch auch verschiedentlich darauf hin, dass bestimmte Teilklassen von Adverbien auch in adnominaler Verwendung auftreten können (vgl. Blatz 1896, S. 39; Behaghel 1989, S. 20; Curme 1922, S. 338 f.; Zifonun/Hoffmann/Strecker 1997, S. 54; Eisenberg 2004, S. 213; Duden-Grammatik 2009, S. 801, 834). ${ }^{3}$ Dieser Verwendungstyp ist zwar nicht prototypisch für Adverbien der betreffenden Klassen, er ist aber auch nicht marginal in dem Sinne, dass er sich nur in bestimmten Textsorten oder Registern findet oder zwangsläufig zu Ausdrücken von zweifelhafter Grammatikalität führt. Auch beschränkt sich die Attribuierbarkeit von Adverbien keineswegs auf Nomina, die ebenso wie Verben Ereignisse bezeichnen. Die Beispiele in (1) dokumentieren diese Verwendungsmöglichkeit anhand der deiktischen Adverbien mit den Bedeutungen ,hier' und ,heute für das Deutsche sowie für vier weitere europäische Sprachen, ${ }^{4}$ die uns im Folgenden als Kontrastsprachen dienen. ${ }^{5}$
DEU Unser Haus hier ist schön.
Das Treffen beute interessiert mich nicht.
ENG Our house here is nice.
The meeting today is of no interest to me.
FRA Notre maison ici est jolie.
La rencontre aujourd'bui ne m'intéresse pas.
POL Nasz dom tu jest ładny.
To spotkanie dzisiaj nie interesuje mnie.
UNG Ez a ház itt szép.
Ez a találkozás ma nem érdekel.

Die attributive Verwendung von Adverbien unterliegt allerdings einer Reihe von formalen und semantischen Beschränkungen, die kaum dokumentiert sind. Das gilt für die sprachvergleichende Grammatikographie in noch stärkerem Maße als für die einzelsprachbezogene, wo sich zumeist nur Feststellungen dazu finden, welche Teilklassen von Adverbien überhaupt in adverbialer Funktion auftreten können.

3 Zum Englischen vgl. vor allem Biber et al. (1999, S. 548) sowie Huddleston/Pullum (2002, S. 446), wo jedoch die fraglichen Adverbien als Präpositionen (ohne Komplement) klassifiziert werden. Zum Französischen vgl. Grevisse (1993, S. 1346), Riegel/Pellat/Rioul (1996, S. 382).

4 Dies sind die Kontrastsprachen im Projekt „Grammatik des Deutschen im europäischen Vergleich“.

5 Die folgenden Abkürzungen werden im Text verwendet: $\mathrm{DEU}=$ Deutsch, $\mathrm{ENG}=$ Englisch, FRA $=$ Französisch, $\mathrm{POL}=$ Polnisch, $\mathrm{UNG}=$ Ungarisch, $\mathrm{ADJ}=$ Adjektiv, $\mathrm{ART}=$ Artikel, DEL $=$ Delativ, DEM $=$ Demonstrativpronomen, GEN $=$ Genitiv, $\mathrm{N}=$ Nomen, PART $=$ Par tizip, POSS $=$ Possessivsuffix, PRÄS $=$ Präsens, $S G=$ Singular, $\mathrm{SUB}=$ Sublativ. 
Vergleicht man die betreffenden Strukturen mit denen benachbarter europäischer Sprachen, zeigen sich grammatische Unterschiede bezüglich der formalen Realisierungsmöglichkeiten von adverbialen Attributen, die ihrerseits helfen, die Perspektive auf den Phänomenbereich im Deutschen zu erweitern: So ist die Juxtaposition attributiver Adverbien - anders als im Deutschen - im Französischen und Polnischen eher beschränkt und im Ungarischen so gut wie gar nicht möglich: Im Französischen werden attributive Adverbien präferiert mit einer formalen Präposition verknüpft, im Polnischen und Ungarischen müssen sie zu diesem Zweck adjektiviert werden. Dieses übereinzelsprachlich feststellbare Spektrum von Anbindungsmöglichkeiten findet sich aber auch - in unterschiedlichem Umfang - in den Einzelsprachen (vgl. DEU der Vortrag gestern vs. der Vortrag von gestern vs. der gestrige Vortrag). Sinnvoll ist es daher, auch in einer einzelsprachbezogenen Analyse verschiedene Verfahren der Anbindung unter einem erweiterten Begriff von adverbialer Modifikation in den Blick zu nehmen.

Im Folgenden werden wir uns dem skizzierten Phänomenbereich aus einer sprachvergleichenden Perspektive zuwenden, dabei aber das Deutsche fokussieren. Auch werden wir nicht alle Vergleichssprachen mit gleicher Ausführlichkeit untersuchen; je nach Problem oder Datenbereich werden einige Sprachen eher in den Vordergrund, andere eher in den Hintergrund rücken. Auch geht es uns nicht um eine möglichst vollständige Beschreibung der Attributionsmöglichkeiten in den einzelnen Sprachen, sondern darum, auf übereinzelsprachliche Uniformitäten und relevante Unterschiede hinzuweisen.

Der Aufsatz gliedert sich wie folgt: In Abschnitt 2 grenzen wir den Untersuchungsbereich auf die Gruppe der temporalen und lokalen Adverbien ein, die wir mit Blick auf ihre adnominalen Verwendungsmöglichkeiten untersuchen werden. In Abschnitt 3 werden die verschiedenen formalen Anbindungsstrategien vorgestellt, von denen Sprachen Gebrauch machen, um Adverbien attributiv verwenden zu können. Gezeigt wird, welche Strategien in welchen Sprachen möglich sind und welche der möglichen Strategien jeweils dominant sind. Abschnitt 4 diskutiert dann drei semantische Restriktionen bzw. Effekte, die für bestimmte Anbindungsstrategien charakteristisch sind: temporale und lokale Kompatibilität (Abschnitt 4.1), Persistenz (Abschnitt 4.2) und Oppositivität (Abschnitt 4.3). Eine Zusammenfassung der Ergebnisse enthält Abschnitt 5.

\section{Temporale und lokale Adverbien}

Die adnominale Verwendung von Adverbien ist weitgehend auf temporale und lokale Adverbien beschränkt. ${ }^{6}$ Wir betrachten im Folgenden ausschließ-

Zum Deutschen vgl. Blatz (1896, S. 39), Curme (1922, S. 339), zum Englischen Huddleston/ Pullum (2002, S. 446), zum Französischen Riegel/Pellat/Rioul (1996, S. 382). Payne/Hud- 
lich Elemente aus diesen Klassen, wobei Adverbien mit quantifizierender (z.B. immer, überall) oder anaphorischer Bedeutungskomponente (daraufbin, daneben) unberücksichtigt bleiben. Tabelle 1 listet die für uns relevante Auswahl für die Vergleichssprachen auf.

\begin{tabular}{|l|l|l|l|l|}
\hline Deutsch & Englisch & Französisch & Polnisch & Ungarisch \\
\hline jetzt & now & maintenant & teraz & most \\
\hline heute & today & aujourd'hui & dzisiaj & ma \\
\hline damals & then & à l'époque & wtedy & akkor \\
\hline gestern & yesterday & hier & wczoraj & tegnap \\
\hline morgen & tomorrow & demain & jutro & holnap \\
\hline
\end{tabular}

Tab. 1: Temporale Adverbien

Die lokalen Adverbien lassen sich in die Gruppe der topologischen und der dimensionalen Adverbien untergliedern (vgl. Klein 2001, S. 583). Topologische Adverbien sind Wörter wie bier, dort und da. Mit ihnen werden Raumabschnitte nach den Parametern der Nähe und Ferne zum Sprecher oder zu einem anderen Kommunikanten bezeichnet. Dimensionale Adverbien sind dagegen Wörter wie links, oben und vorn, mit denen der Sprecher Raumabschnitte unter Einbeziehung der drei räumlichen Dimensionen herausgreifen kann. Der von uns untersuchte Bestand von lokalen Adverbien umfasst die in Tabelle 2 aufgelisteten Ausdrücke. Unberücksichtigt bleiben Adverbien mit Richtungsbedeutung (z.B. bierbin, dorther).

Unter den lokalen und temporalen Adverbien findet sich eine Reihe von Ausdrücken, die formal den Charakter von PPs haben: ENG on the left/ on the right, on top, at the top, at the bottom, at/ in the back, FRA a l'époque, à gauchel à droite, en haut/en bas, POL na lewo/na prawo, na górze/ na dole, z.przodu, na przodzie, z tytu, $w$ tyle. Alle diese Ausdrücke sind mehr oder weniger stark lexikalisiert, und da Lexikalisierung ein graduelles Phänomen ist, verhalten sie sich in syntaktischer wie semantischer Hinsicht teils wie einfache Wörter, teils wie syntaktische Phrasen. ${ }^{7}$ Formal sichtbar wird der defektive phrasale Charakter in den Artikelsprachen am Fehlen des Binnenartikels in Ausdrücken wie ENG on top, in front und FRA à gauche/à droite. (FRA en kombiniert

dleston/Pullum (2010) zeigen jedoch, dass im Englischen auch Satz- und Bereichsadverbien adnominal fungieren können, vgl. the support financially of the local community (ebd., S. 45). Wir gehen auf diese Fälle hier nicht ein.

Uneindeutig ist der Status der Ausdrücke at/in the back sowie at the top/bottom, da die unten angeführten formalen Tests einerseits für eine Kategorisierung als PP sprechen, andererseits das eingebettete Substantiv aber nicht erfragbar ist, vgl. (i) A: Please store your luggage at the back. B: * At which back?/ Where? oder (ii) * At which back/ Where did you say that I can store my luggage? 
grundsätzlich nicht mit Artikeln.) Da die Präposition Teil eines komplexen Wortes ist, wäre zu erwarten, dass sie in Koordinationen nicht ausgelassen werden kann. Bei den polnischen phrasalen Adverbien ist die Weglassbarkeit tatsächlich blockiert (vgl. (2a)), im Unterschied zu syntaktisch regulären PPs (vgl. (2b)).

\begin{tabular}{|c|c|c|c|c|}
\hline Deutsch & Englisch & Französisch & Polnisch & Ungarisch \\
\hline hier & here & ici & tu, tutaj & itt \\
\hline dort, da & there & là-bas & $\operatorname{tam}$ & ott \\
\hline links & on the left & à gauche & na lewo & balra \\
\hline rechts & on the right & à droite & na prawo & jobbra \\
\hline oben & $\begin{array}{l}\text { above, at the } \\
\text { top, on top, } \\
\text { upstairs }\end{array}$ & $\begin{array}{l}\text { dessus, } \\
\text { en haut }\end{array}$ & na górze & fent \\
\hline unten & $\begin{array}{l}\text { at the bottom, } \\
\text { downstairs }\end{array}$ & $\begin{array}{l}\text { dessous, } \\
\text { en bas }\end{array}$ & na dole & lent \\
\hline vorn & in front & devant & $\begin{array}{l}\text { z przodu, } \\
\text { na przodzie }\end{array}$ & elől \\
\hline hinten & $\begin{array}{l}\text { behind, } \\
\text { at/in the back }\end{array}$ & derrière & z tyłu, w tyle & hátul \\
\hline
\end{tabular}

Tab. 2: Topologische und dimensionale Adverbien

(2) POL
a. na górze $\mathrm{i} *$ (na) dole ,oben und unten'
b. na stole i (na) krześle ,auf dem Tisch und (auf) dem Stuhl ${ }^{6}$

Für das Französische ist dieser Koordinationstest nicht anwendbar, da die Auslassung von $a ̀$, de und $e n$ wie in (3) von vielen Sprechern auch für PPs als ungrammatisch beurteilt wird (vgl. Cabredo Hofherr in diesem Band). Im Englischen dagegen kann die Präposition auch bei koordinierten phrasalen Adverbien, die einen Binnenartikel haben, ausgelassen werden (vgl. (4)).

(3) FRA à la mère et la fille

(4) ENG a. on the left and the right

b. at the back and the front

Semantisch zeigt sich der lexikalisierte Status der phrasalen Adverbien darin, dass eine attributive Erweiterung des eingebetteten Nomens entweder zur Ungrammatikalität führt (vgl. (5a)) oder eine konkrete Bedeutung des 
Nomens induziert (vgl. (6)). Eine Ausnahme bildet die Attribuierung durch far im Englischen, wobei die Inakzeptabilität des entsprechenden mit near gebildeten Ausdrucks zeigt, dass das Muster nicht produktiv ist (vgl. (5b)).

(5) ENG a. *the car on the near left

b. the car on the far left

(6) FRA à l'époque antérieure

Analoge Effekte treten auf, wenn die Präposition oder der Binnenartikel durch Wörter derselben Kategorie kommutiert werden (vgl. (7) und (8)).

(7) ENG a. flowers on $/ *_{\text {in }} / *_{\text {at }} / *_{\text {next }}$ to the left

b. the people at the/this back

(8) FRA le discours à l'époque/cette époque

Schließlich ist das eingebettete Nominal in keinem der Fälle referenziell, da es weder erfragt - auf eine Äußerung von à l'époque wie in (9a) kann nicht mit einer Frage wie in (9b) reagiert werden - noch durch ein Relativpronomen pronominalisiert werden kann (vgl. (10)).

(9) FRA a. J'habitais à Rennes à l'époque.

b. À quelle époque est-ce que tu habitais à Rennes?

(10) POL na dole, *który wörtl.: ,im unteren Teil, der

FRA en bas, *qui wörtl. ,im unteren Teil, der

\section{Die Anbindungsstrategien}

\subsection{Juxtaposition}

Um ein Adverb attributiv auf einen nominalen Kopf zu beziehen, stehen übereinzelsprachlich verschiedene Verfahren zur Verfügung. Das formal einfachste Verfahren ist die Juxtaposition, bei der das Adverb adjazent zum nominalen Kopf steht und morphologisch unverändert bleibt. Juxtaponierte Adverbien treten im Deutschen und Englischen sowie z.T. auch im Französischen und Polnischen auf und zwar stets postnominal (vgl. (1)); die im Englischen mögliche Voranstellung von Formen wie in upstairs neighbour behandeln wir als einen Fall von Adjektivierung (Konversion).

\subsection{Adjektivierung}

Adjektivierung ist die zweite mögliche Anbindungsstrategie. Hierbei wird ein Adverb in ein Wort derjenigen Wortart überführt, deren Elemente charakteristischerweise adnominal (attributiv) fungieren. Allerdings werden wir den 
Begriff der adjektivischen Anbindungsstrategie weiter fassen und auch Wörter berücksichtigen, die keine Adjektivierungen im üblichen Sinne sind. Als übergreifenden Terminus wählen wir zu diesem Zweck den Ausdruck adjektivische Entsprecbung. Die Tabellen 3 und 4 führen die adjektivischen Entsprechungen der temporalen und lokalen Adverbien aus den Tabellen 1 und 2 auf.

\begin{tabular}{|l|l|l|l|l|l|}
\hline & Deutsch & Englisch & Französisch & Polnisch & Ungarisch \\
\hline ,jetzt & jetzig & - & - & teraźniejszy & mostani \\
\hline ,heute $^{6}$ & heutig & (now) & - & dzisiejszy & mai \\
\hline ,damals & damalig & (then) & - & - & akkori \\
\hline ,gestern' $^{6}$ & gestrig & - & - & wczorajszy & tegnapi \\
\hline ,morgen & morgig & - & - & jutrzejszy & holnapi \\
\hline
\end{tabular}

Tab. 3: Adjektivische Entsprechungen temporaler Adverbien

\begin{tabular}{|l|l|l|l|l|l|}
\hline & Deutsch & Englisch & Französisch & Polnisch & Ungarisch \\
\hline ,hier & hiesig & - & - & tutejszy & itteni \\
\hline ,dort & dortig & - & - & tamtejszy & ottani \\
\hline ,links & linke & left & - & lewy & bal \\
\hline ,rechts & rechte & right & - & prawy & jobb \\
\hline ,oben` & $\begin{array}{l}\text { obige, } \\
\text { obere }\end{array}$ & $\begin{array}{l}\text { above, } \\
\text { upstairs, top }\end{array}$ & (supérieur) & górny & $\begin{array}{l}\text { fenti, } \\
\text { felső }\end{array}$ \\
\hline ,unten` & untere & $\begin{array}{l}\text { downstairs, } \\
\text { bottom }\end{array}$ & (inférieur) & dolny & $\begin{array}{l}\text { lenti, } \\
\text { alsó }\end{array}$ \\
\hline ,vorn` & vordere & front & (antérieur) & przedni & elülső \\
\hline ,hinten & hintere & back & (postérieur) & tylny & hát(ul)só \\
\hline
\end{tabular}

Tab. 4: Adjektivische Entsprechungen lokaler Adverbien

Zu den adjektivischen Entsprechungen zählen zunächst explizite Ableitungen von adverbialen Stämmen mithilfe von Derivationsaffixen wie DEU -ig, POL $-s\{y,-n y$, UNG $-i$, wobei die Frage der Produktivität für uns keine Rolle spielt. Dabei können auch Stammvarianten auftreten: POL tera $\rightarrow$ teraźniejs:y, tutaj $\rightarrow$ tutejsay, UNG most $\rightarrow$ mostani, ott $\rightarrow$ ottani sowie DEU damals $\rightarrow$

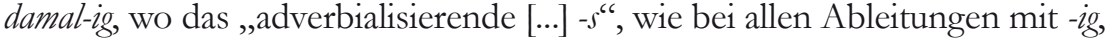
weggelassen wird (Fleischer/Barz 1992, S. 258). Als Ableitungen lassen sich auch Bildungen wie DEU jetrig und biesig betrachten, die auf diachron frühere Formen (iez/ieze bzw. hie) von jetzt und hier zurückgehen (vgl. DWB, 
Bd. 10, Sp. 1320 und 2314). Ein weiterer Fall sind Ableitungen auf der Basis von synchron nicht mehr auftretenden Partikeln, aus denen auch die betreffenden Adverbien abgeleitet sind. Dies betrifft im Deutschen einmal das Adjektiv obig sowie alle Adjektive, die mithilfe des -er-Suffixes gebildet sind, das zwar auf den ersten Blick wie das Komparativmorphem aussieht, aber historisch mit diesem nicht verwandt ist, vgl. DEU ob-er-e, vord-er-e etc. (vgl. Wilmanns 1899, S. 431 f., 649 f.). ${ }^{8}$ Die in Klammern aufgeführten lokalen Adjektive des Französischen lassen sich als lexikalische Entsprechungen solcher Pseudokomparative auffassen, deren lateinische Basis analog strukturiert ist, vgl. FRA supér-ieur $\leftarrow$ LAT sup-er-ior-is etc. Daher sind sie hier mit berücksichtigt, obwohl sie keine Adjektivierungen der korrespondierenden französischen Adverbien sind.

Zur Strategie der adjektivischen Anbindung rechnen wir auch die Möglichkeit, den nominalen Kern eines phrasalen Adverbs zu adjektivieren. Dies betrifft Adjektiv-Adverb-Paare wie ENG top - on top, front - in front, POL przedni - zproodu/na przodzie (,vorn'), górny - na górze (,oben'). Wir behaupten jedoch nicht, dass das phrasale Adverb dem Adjektiv derivationell zugrundeliegt, also den ,Input eines Adjektivierungsprozesses bildet. Vielmehr scheint das Adjektiv direkt aus dem Nomen, das den lexikalischen Kern des phrasalen Adverbs bildet, abgeleitet zu sein. Im Englischen geschieht dies via Konversion, im Polnischen via Derivation. Für das Englische lässt sich alternativ annehmen, dass top, bottom, front, back als Nomen attributiv verwendet werden, so etwa das OED (2011), das diese Elemente teils als adjektivisch verwendete Nomina, teils als adjektivische Bestandteile von Komposita klassifiziert. Collins COBUILD (2006) behandelt sie als Adjektive. Ein Indiz für eine Zuordnung zu den Adjektiven könnte die Kombinierbarkeit mit -most abgeben (vgl. topmost, frontmost etc.), das auf adjektivische Stämme festgelegt zu sein scheint (vgl. *northmost vs. northernmost, Collins COBUILD 2006). Wie das Ableitungsverhältnis zwischen den englischen Adjektiven left/ right und den nominalen Kernen der phrasalen Adverbien on the left/ on the right zu fassen ist, lassen wir offen.

Auch die ungarischen Adjektivbildungen auf -só/-só (felsö,obere') können jeweils auf einen nominalen Kern, z.B. ${ }^{*} f e l$, oberer Teil', zurückgeführt werden, der - erweitert durch synchron nicht mehr produktive Lokalkasussuffixe - in den semantisch äquivalenten Adverbien (fent, oben') enthalten ist.

Wie oben erwähnt betrachten wir im Englischen Wörter wie above, upstairs, downstairs sowie now und then als Adjektive, wenn sie pränominal auftreten (vgl. (11a)), und nehmen an, dass sie via Konversion aus ihren adverbialen Gegenstücken hervorgegangen sind (vgl. (11b)).

Zum Verhältnis zwischen lokalen und temporalen Adjektiven und Adverbien im Deutschen vgl. auch Eisenberg (2002).

9 Die Verwendungsmöglichkeiten von pränominalem now und then sind allerding stark beschränkt, so dass wir diese Wörter im Folgenden nicht weiter berücksichtigen. 
(11) ENG a. the downstairs/upstairs neighbour

b. the neighbour downstairs/upstairs

Quirk et al. (1985, S. 453) sowie Huddleston/Pullum (2002, S. 530 f.) behandeln prä- und postnominale Attribute als Ausdrücke der gleichen Kategorie; erstere als Adverbien, letztere als PPs. ${ }^{10}$ Eine Zuordnung der pränominalen Formen zu den Adjektiven ist nicht nur distributionell, sondern vor allem semantisch motivierbar. Wie in den Abschnitten 4.2 und 4.3 gezeigt wird, verhalten sie sich semantisch nicht wie ihre adverbialen Gegenstücke, sondern eher wie Adjektive; der Kontrast zwischen den voran- und nachgestellten Elementen in Paaren wie upstairs neighbour - neighbour upstairs ist analog zu dem zwischen Adjektiven und phrasalen Adverbien in Paaren wie ENG top drawer - drawer on top oder left door - door on the left etc.

Betrachten wir zuletzt noch zwei topologische Adjektive, die ihrerseits die Basis für die adverbialen Gegenstücke bilden. Dies sind die Adjektive linke/ rechte im Deutschen und ihre Äquivalente lewy/prawy im Polnischen sowie bal/jobb im Ungarischen; aus ihnen sind - wie in Tabelle 2 verzeichnet die Adverbien DEU links/rechts, POL (na) lewo/(na) prawo und UNG balra/ jobbra abgeleitet. Im Deutschen gehen die Adverbien auf eine Genitivform des Adjektivs zurück (vgl. DWB, Bd. 12, Sp. 1049), im Polnischen sind sie mithilfe des produktiven Adverbialisierungssuffixes -o gebildet, im Ungarischen mithilfe des Kasussuffixes - $r A$, das synchron als Kasussuffix mit direktionaler Bedeutung produktiv ist (vgl. Budapestre, nach Budapest').

\subsection{Formale Verknüpfung}

Die dritte von uns untersuchte Anbindungsstrategie ist die formale Verknüpfung, bei der das Adverb mithilfe eines formalen, semantisch leeren Verknüpfungselements auf den nominalen Kopf bezogen wird. In den Vergleichssprachen treten als solche formalen Verknüpfer vor allem Präpositionen auf: DEU von, ENG of, FRA de (vgl. (12)); im Englischen findet sich mit dem Marker für den so genannten ,sächsischen Genitiv zudem ein Klitikon in dieser Funktion (vgl. (13)).

DEU [...] während er Tiefkühlkost oder das Essen von gestern aufwärmt [...] (DeReKo)

10 Üblich ist eine Zuordnung zu den Adverbien, vgl. auch Biber et al. (1999, S. 575). Bauer (1983, S. 206) diskutiert die Möglichkeit von Adverb-Nomen-Komposita im Englischen und schreibt in diesem Zusammenhang: ,The only recent example of this pattern I have noted is now generation, where now could alternatively be interpreted as an adjective formed by conversion." Bolinger (1967, S. 11) deutet an, dass mit Blick auf attributiv verwendete Adverbien auch von „adverbs doubling as adjectives or vice versa“ gesprochen werden könne. Collins COBUILD (2006) klassifiziert die pränominalen Attribute durchgehend als Adjektive, ebenso - bis auf backstage - das OED (2011). 
ENG Whether the steep falls of yesterday turn into something more serious remains to be seen. (BNC)

FRA Ce matin, revenant sur notre discussion d'hier, il m'a expliqué d'une façon charmante comment il comprenait ma position [...] (FRANTEXT)

(13) ENG Today's meeting comes after a weekend in which [...] (BNC)

Bei den präpositionalen Verknüpfern ist entscheidend, dass sie in der hier intendierten Verwendung keine inhaltliche, lokale oder temporale Bedeutung haben; für Präpositionen wie DEU von, ENG of, FRA de heißt das, dass sie als formale Verknüpfer nicht ablativisch gelesen werden. So wäre etwa die Präposition von in der Lichteinfall von oben kein formaler Verknüpfer, weil der Ausdruck nicht im Sinne von, der Lichteinfall, der oben ist ${ }^{6}$, sondern nur - ablativisch - im Sinne von, der Lichteinfall, der von oben kommt ${ }^{6}$ gelesen werden kann. Analoges gilt für eine weitere Anbindungsstrategie, die wir hier nur der Vollständigkeit halber erwähnen, auf die wir aber im Folgenden nicht weiter eingehen, nämlich die Anbindung durch Einbettung in eine Partizipialphrase oder einen Relativsatz. Diese Strategie findet sich unter den Vergleichssprachen vor allem im Ungarischen, das Partizipialphrasen einsetzt, deren verbale Köpfe zumeist Formen von Existenz- und Positionsverben sind und deren inhaltlicher Beitrag zur Bedeutung der gesamten Phrase eher zweitrangig ist (vgl. (14)).

$\begin{array}{llll}\text { UNG } & \text { a lent lévő } & \text { link } \\ & \text { die unten } & \text { sein.PARTPRÄS } & \text { Link } \\ & \text { wörtl. ,der unten seiende Link }\end{array}$

\subsubsection{Verteilung der Anbindungsstrategien in den Vergleichssprachen}

Eine Übersicht über das Auftreten der Anbindungsstrategien in den Vergleichssprachen gibt Tabelle 5 .

\begin{tabular}{|l|l|l|l|}
\hline & Juxtaposition & Adjektivierung & Formale Verknüpfung \\
\hline DEU & + & + & $(+)$ von \\
\hline ENG & + & + & + of, s \\
\hline FRA & $(+)$ & - & + de \\
\hline POL & $(+)$ & + & - \\
\hline UNG & - & + & - \\
\hline
\end{tabular}

Tab. 5: Die Anbindungsstrategien in den Vergleichssprachen 
In drei der Vergleichssprachen kommen bestimmte Strategien überhaupt nicht vor $(,,-")$, andere sind eher dispräferiert $\left(,(+)^{"}\right)$. So gibt es im Ungarischen keine formalen Verknüpfer für attributive Adverbien; solche existieren dort aber auch nicht für attributive Nominal- oder Adpositionalphrasen. Possessivattribute werden affixal durch Kopfmarkierung angebunden (vgl. az igazgató autója (ART Direktor Auto.POSS3SG), das Auto des Direktors'), attributive Adpositionalphrasen durch Adjektivierung der Adposition mit dem Suffix -i (vgl. a polc mögötti könyvek. (ART Regal hinter.ADJ Bücher) ,die Bücher hinter dem Regal'). Die Adjektivierung ist damit generell eine der dominanten Anbindungsstrategien im Ungarischen. Die Juxtaposition ist zwar möglich, allerdings in einem so eingeschränkten $\mathrm{Maß}$, dass wir sie hier vernachlässigen. Laczkó (2003, S. 213) führt als Beispiel (15a) an; viele Muttersprachler beurteilen solche Strukturen allerdings als problematisch. Akzeptabler werden sie, wenn das Nomen von einem Demonstrativum determiniert wird (vgl. (15b)), was im Übrigen auch für die analogen Strukturen im Französischen und Polnischen gilt. Steht das Nomen jedoch in einem semantischen Kasus, ist die Juxtaposition - ebenso wie bei attributiven Nominal- und Adpositionalphrasen - ausgeschlossen (vgl. (15c)).

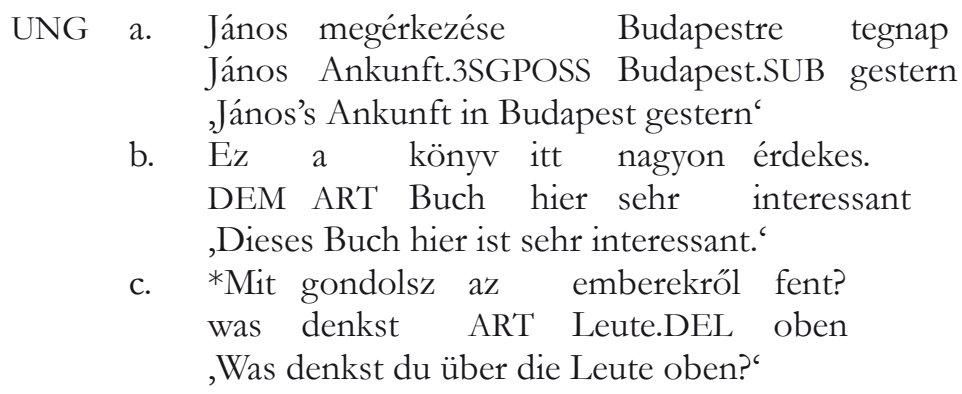

Auch im Polnischen ist die Adjektivierungsstrategie dominant, Juxtaposition eher beschränkt und formale Verknüpfung ausgeschlossen. Formale Verknüpfer gibt es auch zur attributiven Anbindung von Nominalen nicht; diese werden entweder als Genitivattribute realisiert, vgl. dzień pracy (Tag Arbeit.GEN) ,Arbeitstag', oder durch Adjektivierung in relationale Adjektive überführt, vgl. sok. jabtkony (Saft $\left.\mathrm{Apfel}_{\mathrm{ADJ}}\right)$, Apfelsaft'. Die Adjektivierung spielt auch hier generell eine zentrale Rolle im Bereich der Attribution.

Nahezu spiegelbildlich zum Ungarischen und Polnischen verhält sich das Französische, wo die formale Verknüpfung die dominante Strategie ist, während Adjektivierung gar nicht und Juxtaposition ebenfalls nur beschränkt auftritt. Die Präposition de, die als formaler Verknüpfer fungiert, ist auch für die Anbindung von nominalen Attributen ein zentrales Mittel, sei es zur Realisierung von Argumenten (vgl. le vol de documents), sei es zur Bildung kompositaähnlicher N-de-N-Strukturen (vgl. la chemise de nuit, Wandruszka 1972). 
Im Deutschen und Englischen treten alle drei Anbindungsstrategien auf; allerdings ist die formale Verknüpfung im Deutschen eher marginal. Das Englische verfügt dagegen mit dem sächsischen Genitiv über einen zusätzlichen Verknüpfer, der auch zur Bildung von kompositaähnlichen Syntagmen eingesetzt wird (vgl. men's wear). Zudem spielt die Präposition of im Englischen eine wichtigere Rolle als von im Deutschen und ist mit Blick auf ihr Funktionsspektrum eher mit dem französischen de vergleichbar: Auch sie dient zur Bildung kompositaähnlicher Strukturen (vgl. bird of prey, Klinge 2005) sowie daneben zur Anbindung von nominalen Argumenten (vgl. the theft of the documents) und Possessivattributen (vgl. the trunk of the car).

\section{Semantische Restriktionen und Effekte bei den Anbindungsstrategien}

Die Anbindungsstrategien unterliegen in den Vergleichssprachen in unterschiedlichem Maße semantischen Beschränkungen; wo verschiedene Strategien möglich sind, sind diese oft mit unterschiedlichen semantischen Effekten verknüpft. Übereinzelsprachlich zeichnet sich eine Korrelation zwischen Form und Funktion ab, wobei die Juxtaposition tendenziell mit dem einen, die Adjektivierung und formale Verknüpfung tendenziell mit dem anderen Bedeutungstyp korreliert.

\subsection{Temporale und lokale Kompatibilität}

Juxtaponierte temporale und lokale Adverbien müssen mit anderweitig im Satz kodierten temporalen und lokalen Bestimmungen vereinbar sein. Diese Beschränkung gilt zum einen Teil auch für andere Temporalattribute, zum anderen Teil ist sie für juxtaponierte Adverbien spezifisch und findet sich dann nicht für adjektivische oder formal verknüpfte Attribute. Wir beginnen zunächst mit den temporalen Adverbien und fahren dann mit den lokalen fort. Da die semantischen Kontraste, die es zu erfassen gilt, äußerst subtil sind und die Sprecherurteile häufig schwanken, werden wir die Diskussion in den nächsten beiden Abschnitten hauptsächlich am Deutschen führen.

\subsubsection{Temporale Adverbien}

Für die Frage der Attribuierbarkeit temporaler Adverbien ist die Unterscheidung zwischen ereignisbezeichnenden (vgl. Vortrag, Spiel, Aufmarsch etc.) und gegenstandsbezeichnenden (vgl. Hut, Wobnung, Zeitung etc.) Nomina relevant. Ereignisse sind nach unserer Terminologie Entitäten, die eine zeitliche Ausdehnung haben; sie können statisch oder dynamisch sein. Gegenstände sind dagegen Entitäten, die in der Zeit konstant bleiben. ${ }^{11}$

11 Vgl. die Abgrenzung von ,first-order nominals‘ gegenüber, second-order nominals‘ in Lyons (1977, S. 446). 


\subsubsection{Ereignisbezeichnende Nomina}

Wie die Beispiele in (16) zeigen, kann die Grammatikalität von Sätzen davon abhängen, ob der Zeitbezug eines Temporalattributs mit dem Satztempus vereinbar ist. ${ }^{12}$ Die diesbezügliche Kotemporalitätsbeschränkung ist bei ereignisbezeichnenden Nomina z.T. unabhängig von der Form des Attributs. Sie gilt gleichermaßen für juxtaponierte und adjektivische Attribute sowie für Relativsätze, die das Kopfnomen mithilfe eines Temporaladverbs zeitlich situieren (vgl. (17)). ${ }^{13}$ Für formal verknüpfte Attribute ist sie eher auf Fälle wie (16) beschränkt.
a. Das Spiel gestern wurde/*wird von vielen Zuschauern gesehen. ${ }^{14}$
b. Der NPD-Aufmarsch gestern wurde/*wird von Gegen- demonstranten gestört.
c. Die Veranstaltung gestern endete/*endet gegen $22 \mathrm{Uhr}$
a. Das Spiel, das gestern stattfand, wurde/*wird von vielen Zuschauern gesehen.
b. Der gestrige NPD-Aufmarsch wurde/*wird von Gegen- demonstranten gestört.
c. Die Veranstaltung von gestern endete/*endet gegen 22 Uhr.

Unmittelbar nachvollziehbar ist die Beschränkung im Zusammenhang mit Wahrnehmungs-, Manipulations- und Phasenverben (vgl. (16a-c), (17a-c)): Ereignisse, die beobachtet oder manipuliert werden, müssen zeitgleich mit der Beobachtung bzw. Manipulation ablaufen, zeitliche Phasen eines Ereignisses liegen naturgemäß innerhalb der Ereigniszeit. Konzeptuell nicht zwingend wäre die geforderte Kotemporalität bei Verben und Prädikativa, die Bewertungen ausdrücken; dennoch finden wir sie auch hier (vgl. (18a, b)):
a. Der Vortrag gestern gefiel/*gefällt mir überhaupt nicht.
b. Die Aufführung gestern war $/ *$ ist umwerfend.

Allerdings sind die Sprecherbeurteilungen von Sätzen wie (18b) durchaus schwankend, gleiches gilt für Sätze wie (19a, b).
a. ? Ansonsten sind Dortmunds Eckstöße gestern über- ragend. (Hörbeleg, Sportschau, 05.03.2011)
b. ? Der Vortrag gestern ist der schlechteste, den er jemals gehalten hat.

\footnotetext{
12 Vgl. schon Ulvestad (1972, S. 180, Fn. 55).

13 In diesem Fall muss natürlich das Tempus des Prädikatsverbs mit dem Zeitbezug des Adverbs kompatibel sein.

14 Die Sätze sind grammatisch, wenn man das Tempus als historisches Präsens liest. Diese Lesart schließen wir im Folgenden aus.
} 
Drückt die Prädikation des Matrixsatzes einen Zustand aus, der sich als kausale Wirkung aus dem in der Vergangenheit situierten Ereignis ergibt, ist die temporale Beschränkung tendenziell aufgehoben. Vereinfacht gesagt: Was die Satzprädikation in Bezug auf das vergangene Ereignis ausdrückt, muss auch für die Gegenwart oder Zukunft von Relevanz sein. Das zeigt sich in Prädikativkonstruktionen wie (20a, b), sowie insbesondere in Beispielen wie (21).
a. Mein Auftritt gestern ist*(mir) total peinlich, ich habe mich sowas von lächerlich gemacht. (Internet)
b. Die Prüfung gestern ist *(für sie) ein Desaster.
a. Die Razzia gestern rückt ihn in die Nähe eines Krimi- nellen. (DeReKo)
b. Der Beschluss gestern schränke Demonstrationen und die Berichterstattung der Presse im Vorfeld der vor- gezogenen Präsidentenwahl weiterhin ein, berichteten georgische Medien. (DeReKo)
c. Unsere Recherche gestern bestätigt diese Einschätzung: Auch tagsüber parken Sattelzüge am Rand von Kaiser- graben und Steinbergstraße. (DeReKo)

Keine Beschränkung gibt es bei Verben, die aufgrund ihrer Bedeutung keine Kotemporalität zwischen dem betreffenden Ereignis und der Satzprädikation erfordern. Dazu gehören Verba dicendi; anders als etwa das Wahrnehmen oder Manipulieren von Ereignissen erfordert das Reden über dieselben nicht deren zeitliche Präsenz (vgl. (22)).

(22) DEU a. Ihr Vortrag gestern wird sehr gelobt.

b. Über das Spiel gestern werden wir morgen sprechen.

Die Beschränkung gilt mutatis mutandis auch für die von uns betrachteten Kontrastsprachen; wir verzichten hier aber auf eine Auflistung der relevanten ungrammatischen Beispiele. (23) nennt jeweils ein Beispiel aus dem Englischen und Französischen für Verba dicendi. Für das Französische ist zu beachten, dass alle Beispiele mit juxtaponiertem Adverb in diesem Zusammenhang von unseren Informanten als ungrammatisch beurteilt wurden.

(23) ENG

FRA
The confrontation yesterday between Brett and Martin will be talked about for years. (Anderson 1983, S. 12) La confrontation d'hier entre Brett et Martin fera l'object de commentaire pendant des a années.

\subsubsection{Gegenstandsbezeichnende Nomina}

Die Kotemporalitätsbeschränkung gilt auch bei gegenstandsbezeichnenden Nomina für die betreffenden Typen von Verben, vgl. etwa (24). In analoger 
Weise sind auch die Prädikativkonstruktionen in (25) akzeptabler, da hier die Gültigkeit der Satzprädikation nicht auf den durch das Temporaladverb bezeichneten Zeitrahmen beschränkt ist.

a. Die Schiffe gestern sahen/*sehen wir sehr deutlich.

b. Dein Kleid gestern gefiel/*gefällt mir gar nicht.

a. Mein Kleid gestern ist*(mir) total peinlich.

b. Ich denke die Leute gestern sind froh, dass unser Bürgermeister Tempelhof endlich vom Flugverkehr befreit hat. (Internet)

Unterschiede zur Klasse der ereignisbezeichnenden Nomina zeigen sich in Fällen, in denen nicht-kotemporale Adverbien bei ereignisbezeichnenden Nomina zugelassen sind, bei gegenstandsbezeichnenden dagegen nicht. So sind die Sätze in (26), in denen das Temporaladverb ein gegenstandsbezeichnendes Nomen modifiziert, im Gegensatz zu denen in (21) im Präsens nicht möglich.
a. Der Mann gestern rückte/*rückt ihn in die Nähe eines Kriminellen.
b. Der Mann gestern schränkte/*schränkt die Demonstra- tionsfreiheit ein.
c. Der Mann gestern bestätigte/*bestätigt diese Einschät- zung.

Gegenstände selbst haben keine kausalen Wirkungen, das ist der relevante Unterschied zu den Sätzen in (21). Die entsprechenden Sätze in (26) werden aber bezeichnenderweise dann akzeptabel, wenn sich das Denotat der Nominalphrase metonymisch als Sachverhalt umdeuten lässt, bei (26a) und (26c) etwa im Sinn von „die Sache mit dem Mann gestern“ (vgl. (27a, b)).
a. Der Mann gestern [= die Sache mit dem Mann gestern] rückt ihn in die Nähe eines Kriminellen.
b. Der Mann gestern [= die Sache mit dem Mann gestern] bestätigt diese Einschätzung.

Testbar sind solche Sachverhaltslesarten über die Wahl der Prädikatsverben: Verben, die eine Gegenstandslesart ihres Subjekts oder Objekts erzwingen wie mähen, waschen, darunter auch Positionsverben wie stehen, liegen, sind nicht kompatibel mit Sachverhaltslesarten. Entsprechende Sätze mit juxtaponierten Temporaladverbien sind daher immer ungrammatisch (vgl. (28)).
a. Der Mann gestern *steht/stand drei Stunden lang vor der Tür.
b. Das Hemd gestern *wird/wurde gründlich gewaschen.
c. Der Rasen gestern *wird/wurde später gemäht. 
Umgekehrt können Sätze wie (29a) grammatisch sein, in denen das Verb eine Ereignislesart seines Subjekts erzwingt, dieses aber durch ein gegenstandsbezeichnendes Nomen (mit juxtaponiertem Temporaladverb) realisiert wird, sofern dessen Denotat wie im vorliegenden Beispiel als Ereignis umgedeutet werden kann. Solche Fälle verhalten sich regulär wie die entsprechenden Sätze mit ereignisbezeichnendem Subjekt (vgl. (29b)). Sie sind grammatisch, da die Satzprädikation eine kausale Wirkung des durch das Subjekt bezeichneten Ereignisses bezeichnet.
a. Der 35-Kilo-Sack gestern steckt mir noch heute in den Knochen. (interpretiert im Sinne von „das Tragen des 35-Kilo-Sacks")
b. Der Vortrag gestern liegt mir noch heute im Magen.

Beispiele wie in (26) werden allerdings dann akzeptabel, wenn das Temporaladverb durch einen formalen Verknüpfer angebunden wird (vgl. (30)); hier zeigt sich ein relevanter Unterschied zwischen den möglichen Anbindungsstrategien.
a. Der Mann von gestern rückt ihn in die Nähe eines Kriminellen.
b. Der Mann von gestern schränkt die Demonstrations- freiheit ein.
c. Der Mann von gestern bestätigt diese Einschätzung.

Entsprechende Kontraste zeigen sich auch in folgenden Beispielen:

DEU a. *Die Zeitung gestern liegt auf dem Tisch.

b. Die Zeitung von gestern liegt auf dem Tisch.

32) ENG a. *The newspaper yesterday is on the table. (Anderson 1983, S. 12)

b. Yesterday's newspaper is on the table.

33) FRA a. *Le journal hier se trouve sur la table.

b. Le journal d'hier se trouve sur la table.

Bei formaler Verknüpfung bleibt der temporale Bedeutungsbeitrag des Attributs außerhalb der NP offenbar semantisch unzugänglich und kann daher nicht mit dem Tempus des Prädikatsverbs konfligieren. Semantisch lässt sich das dahingehend deuten, dass eine durch ein formal verknüpftes Attribut kodierte temporale Eigenschaft mit dem Denotat des Kopfnomens enger oder fester assoziiert ist als eine temporale Eigenschaft, die durch ein juxtaponiertes Attribut ausgedrückt wird. Das zeigt sich auch daran, dass solche durch einen formalen Verknüpfer eingeleiteten Phrasen auch prädikativ verwendbar sind, was generell eine Eigenschaft von Ausdrücken ist, die als qualitative Modifikatoren fungieren (vgl. (34)). Zudem können die Ausdrücke als ganze übertragene Bedeutungen annehmen (vgl. (35)). 
(34) DEU Die Zeitung ist von gestern.

ENG This paper is yesterday's.

FRA Ce journal est d'hier.
a. die Vorstellungen von gestern
b. Ideen von heute
c. die Frau von heute

\subsubsection{Lokale Adverbien}

Der gerade beschriebene Unterschied zwischen juxtaponierten und formal verknüpften temporalen Adverbien zeigt sich in analoger Weise auch bei den lokalen Adverbien. Die Interpretation von Sätzen wie (36a, b) unterliegt zwei Beschränkungen, die durch das juxtaponierte deiktische Adverb induziert werden. Zum einen muss sich die durch das Subjekt bezeichnete Person oben befinden, und zwar relativ zu dem gegebenen Bezugsraum (z.B. einem Bürogebäude), und zum anderen muss sich der Sprecher ebenfalls innerhalb des Bezugsraums befinden, und zwar im Verhältnis zu der fraglichen Person unten. Die erste Bedingung kann in (36b) nicht erfüllt werden und daher ist dieser Satz ungrammatisch. Die zweite Bedingung ist nicht erfüllt, wenn sich der Sprecher außerhalb des Bezugsraums, also etwa zu Hause befindet, während er über seinen Kollegen im Büro spricht. In solchen Kontexten kann ein Satz wie (36a) nicht geäußert werden. ${ }^{15}$

a. Mein Kollege oben macht wieder Lärm.

b. $\quad *$ Mein Kollege oben steht gerade unten vor der Haustür.

Beide Beschränkungen gelten nun nicht, wenn das Adverb durch eine Präposition angebunden wird (vgl. (37)).

a. Mein Kollege von oben macht wieder Lärm.

b. Mein Kollege von oben steht gerade unten vor der Haustür.

Auch hier lässt sich davon sprechen, dass die fragliche lokale Eigenschaft mit dem Denotat des Kopfnomens enger oder fester assoziiert ist: Sie ist ja auch dann mit dem Gegenstand verknüpft, wenn dieser sich nicht in dem deiktischen Bezugsraum befindet, in Bezug auf den die Interpretation des deiktischen Temporaladverbs festgelegt ist.

15 Möglich ist aber, dass der Sprecher das deiktische Zentrum verschiebt, indem er sich und den Hörer gedanklich in den fraglichen Bezugsraum versetzt, z.B. in Äußerungen wie: Stell dir mal unser Institut vor. Ich sitze in meinem Büro und mein Kollege oben macht wieder Lärm. 


\subsection{Persistenz}

Wie auch immer man den Begriff der fester assoziierten Eigenschaft fasst, relevant ist, dass juxtaponierte Attribute solche Eigenschaften nicht ausdrücken können, während sich der fragliche semantische Effekt sprachabhängig bei bestimmten Teilklassen von formal verknüpften und adjektivischen Attributen findet. Wir zeigen in diesem Abschnitt, dass der Effekt auch bei lokalen Adjektivattributen auftreten kann und sprechen in diesem Zusammenhang von Persistenz bzw. persistenten Eigenschaften.

Persistent ist eine lokale Eigenschaft dann, wenn ein Gegenstand sie nicht nur aufgrund seiner zufälligen momentanen Position hat, sondern wenn sie im jeweiligen Kontext für den Gegenstand charakteristisch ist und daher mit diesem fest assoziiert erscheint. In diesem Sinn beschreibt Bolinger (1967) die Beschränkung für die pränominale, also adjektivische Verwendung von ENG nearby:

The adjective nearby can be used of something stable enough to preempt a location - a nearby building, a nearby group - but not of something that may conceivable move off the next moment: *a nearby man, *a nearby bus. (Bolinger 1967, S. 11)

Die lokalen Eigenschaften von Gegenständen, die sich fest an einer Position befinden, sind persistent und können durch adjektivische oder formal verknüpfte Attribute versprachlicht werden; die Attribution eines juxtaponierten Adverbs ist in diesem Fall auch möglich, sofern die betreffende Sprache Juxtaposition überhaupt zulässt (vgl. (38)).

$\begin{array}{llll}\text { DEU } & \text { die hinteren Sitzplätze } & \text { vs. } & \text { die Sitzplätze hinten } \\ \text { POL } & \text { tylne siedzenia } & \text { vs. } & \text { siedzenia } z \text { tyłu } \\ \text { FRA } & \text { les sièges de derrière } & \text { vs. } & \text { les sièges derrière } \\ \text { ENG } & \text { the back seats } & \text { vs. } & \text { the seats at/in the back }\end{array}$

Bewegliche Gegenstände wie z.B. Personen scheinen dagegen keine persistenten lokalen Eigenschaften zu haben; Beispiele wie die in der linken Spalte von (39), in denen die betreffende Lokalisierung durch ein Adjektiv oder ein formal verknüpftes Adverb ausgedrückt würde, sind inakzeptabel.

$\begin{array}{ll}\text { DEU } & \text { ??die hinteren Leute } \\ \text { POL } & \text { *ylni ludzie } \\ \text { FRA } & \text { *les gens de derrière } \\ \text { ENG } & \text { *the back people }\end{array}$

$$
\begin{array}{ll}
\text { vs. } & \text { die Leute hinten } \\
\text { vs. } & \text { ludzie } z \text { tyłu } \\
\text { vs. } & \text { les gens derrière } \\
\text { vs. } & \text { the people in/at the back }
\end{array}
$$

Es ist allerdings zu beachten, dass die Persistenz oder Nicht-Persistenz einer lokalen Eigenschaft abhängt von der Konzeptualisierung des Gegenstands, dem sie zugeschrieben wird, und damit von der Intension des Kopfnomens. Dies zeigt der Akzeptabilitätsunterschied zwischen Ausdrücken 
wie ??die binteren Leute und die binteren Nachbarn (bzw. Nachbarn von binten): Die Positionen von Nachbarn als Nachbarn sind persistent, die momentanen Positionen von Personen als Personen dagegen nicht.

Schließlich sind auch die lokalen Eigenschaften von Ereignissen nicht persistent, sofern diese nicht iterativ eintreten und somit für eine bestimmte Umgebung nicht charakteristisch sein können, vgl. dazu die Grammatikalitätskontraste in Beispielen wie (40).

$\begin{array}{llll}\text { DEU } & \text { *der obere Streit } & \text { vs. } & \text { der Streit oben } \\ \text { POL } & \text { *oórny spór } & \text { vs. } & \text { spór na górze } \\ \text { FRA } & \text { *la dispute de derrière } & \text { vs. } & \text { la dispute derrière } \\ \text { ENG } & * \text { the upstairs fight } & \text { vs. } & \text { the fight upstairs }\end{array}$

Bedeutungsunterschiede zwischen juxtaponierten und adjektivisch angebundenen Attributen treten auch bei den topologischen Lokalattributen auf. Der Kontrast zwischen den Ausdrücken das Telefonbuch hier vs. das biesige Telefonbuch beruht darauf, dass im ersten Fall ein Gegenstand lokalisiert wird, im zweiten Fall ihm dagegen eine charakteristische Eigenschaft zugesprochen wird, z.B. das Telefonbuch des Ortes zu sein, in dem sich der Sprecher befindet, genauer: auf den sich biesige indexikalisch bezieht. Diese Lesart hat der Ausdruck das Telefonbuch bier nicht, da juxtaponierte Attribute keine persistenten Eigenschaften bezeichnen können. Umgekehrt können topologische Adjektive wie hiesig und dortig im Deutschen nicht demonstrativ, d.h. mit einer Zeigegeste verwendet werden, was darauf hinweist, dass sie ihrerseits ausschließlich persistente Eigenschaften bezeichnen können, vgl. Schau mal, die Frau dort mit dem komischen Hut! vs. ?? Schau mal, die dortige Fran mit dem komischen Hut!

Wie DEU hiesig und dortig verhalten sich auch POL tutejszy und tamtejs:y. Beide können nicht zum Ausdruck von nicht-persistenten lokalen Eigenschaften gebraucht werden, vgl. *tutejsza ksiąंka wörtl., (das) hiesige Buch'vs. ta ksiazkea tutaj, dieses Buch hier'. Möglich ist dagegen tutejsza biblioteka ,die hiesige Bibliothek ${ }^{6}$, da Bibliotheken (in der Regel) einen festen Ort haben.

Dagegen sind die lokalen $i$-Adjektive des Ungarischen nicht auf persistente Eigenschaften festgelegt und verhalten sich in dieser Hinsicht wie juxtaponierte Adverbien in den anderen Vergleichssprachen. Das gilt für dimensionale Adjektive ebenso wie für topologische. Möglich ist daher (41a) - vgl. dagegen (40) - ebenso wie (41b) in der nicht-persistenten Lesart des Adjektivs.

$\begin{array}{llll}\text { UNG a. } & \text { a lenti } & \text { vita } \\ & & \text { ART unten.ADJ } & \text { Streit } \\ & \text {,der Streit unten } & \\ \text { b. } & \text { az itteni } & \text { telefonkönyv } \\ & \text { ART hier.ADJ } & \text { Telefonbuch } \\ & \text {,das hiesige Telefonbuch/das Telefonbuch hier }\end{array}$


Die mit von gebildeten Ausdrücke von bier/von dort drücken ebenfalls persistente Eigenschaften aus. Hier ist die Abgrenzung von abstrakten ablativischen Lesarten im Sinne von ,Ursprung 6 /,Herkunft' schwierig (vgl. die Leute von hier). Beispiele mit nicht-ablativischen Lesarten sind in (42) angegeben. Sprecherurteile schwanken in Bezug auf die Bewertung von Ausdrücken wie in (42b). Eindeutig grammatisch sind Beispiele wie in (42a), in denen das Attribut regulär eine Argumentstelle des Nomens besetzt, die auch durch eine lokativische von-PP gefüllt werden könnte (vgl. das Telefonbuch von Bern).
a. das Telefonbuch von hier, der Bürgermeister von hier, der Stadtplan von hier
b. die Gärten von hier, die Behörden von hier, die Lebens- qualität von hier

Im Englischen können die Lokalattribute of here/ of there ähnlich wie im Deutschen gebraucht werden (vgl. (43)).

(43) ENG a. The mountains of here just jut up out of nowhere. (Internet)

b. $\quad[. .$.$] I would guess the units seen on the streets of here$ are likely US exports [...] (Internet)

Im Französischen ist die formale Verknüpfung von lokalen Adverbien mit de die dominante Strategie (vgl. (44)). Beispiele mit juxtaponierten Adverbien finden sich dennoch (vgl. (45)). Der Anwendungsbereich der formalen Verknüpfung geht zwar weiter als im Deutschen (vgl. la vie d'ici vs. ??das Leben von hier, l'étage du haut vs. ?? die Etage von oben), aber nicht so weit, dass sie auch zufällige Lokalitäten kodieren könnte: FRA l'annuaire téléphonique d'ici kann nur das Telefonbuch von hier bezeichnen. ${ }^{16}$

(44) FRA a. Il n'y a pas cinquante fleurs dans les dix-neuf jardins d'ici. (FRANTEXT)

b. Le climat de là-bas lui convient si bien [...]. (FRANTEXT)

c. Je sais maintenant que ma vie d'ici est fausse [...]. (FRANTEXT)

(45) FRA a. «Les gens ici sont mauvais, change de coin!» (FRANTEXT)

16 Das Deutsche ist mit Blick auf die von-Attribute möglicherweise dahingehend beschränkt, dass in einem Ausdruck der Form $X$ von $Y$ der mit $X$ bezeichnete Gegenstand ein Teil des mit $Y$ bezeichneten räumlichen Bereichs sein muss. Eine solche Teil-Ganzes-Beziehung ist zwar in die Berge von bier gegeben, nicht aber in die Etage von oben, da oben hier nicht auf einen gröBeren Raumbereich verweist, von dem die Etage ein Teil ist. 
b. Colette [...] à qui je demande si elle connait le propriétaire de la jolie maison là-bas, me répond [...] (FRANTEXT)

c. Pour la première fois, je me suis représenté clairement sa vie ici, en dehors de mes visites [...]. (FRANTEXT)

\subsection{Oppositivität}

Die zweite charakteristische Eigenschaft, durch die sich adjektivische und durch formale Verknüpfer angebundene lokale Attribute von juxtaponierten unterscheiden können, bezeichnen wir als Oppositivität. Mit diesem Begriff ist folgendes gemeint:

(46) Sei $\alpha$ ein adnominaler Modifikator, der eine Lokalisierung $l_{1}$ einer räumlichen Dimension $D_{1}$ (Höhe, Breite, Tiefe) benennt, und $\beta$ ein nominaler Kopf, der einen Gegenstand vom Typ $T$ bezeichnet: Dann ist $\alpha$ oppositiv, wenn ein Ausdruck $\gamma$ der Form $\alpha \beta$, wobei $\alpha$ Modifikator von $\beta$ ist, präsupponiert, dass es im referenziellen Kontext von $\gamma$ einen Gegenstand vom Typ $T$ mit einer Lokalisierung $l_{2}\left(\neq l_{1}\right)$ aus der Dimension $D_{1}$ gibt.

Dazu ein Beispiel: Ein Ausdruck wie die obere Scbublade präsupponiert, dass es auch eine untere Schublade gibt, ein Ausdruck wie die linke Tür, dass es auch eine rechte Tür gibt und ein Ausdruck wie die vordere Sitzreihe, dass es auch eine hintere Sitzreihe gibt. Relevant ist in diesem Zusammenhang der jeweilige referenzielle Kontext. Die Existenzpräsupposition gilt nur für einen bestimmten Gegenstand oder räumlichen Bereich; beide sind im jeweiligen referenziellen Kontext über Teil-Ganzes-Beziehungen erschließbar. Bezogen auf die obigen Beispiele heißt das: Präsupponiert wird die Existenz einer weiteren Schublade, Tür, Sitzreihe bezogen auf denjenigen Gegenstand oder räumlichen Bereich, zu dem die genannte obere Schublade, linke Tür oder vordere Sitzreihe gehört. Dass es sich hierbei um eine Präsupposition handelt, zeigt sich darin, dass diese bei Negation erhalten bleibt. In Sätzen wie (47) gilt weiterhin, dass der betreffende Gegenstand oder räumliche Bereich eine Schublade, Tür oder Sitzreihe in der jeweils gleichen Dimension, aber mit anderer Lokalisierung aufweist.
a. Dieser Typ von Kommode hat keine untere Schublade.
b. In diesem Rahmen wurde keine linke Tür eingebaut.
c. Im Seminarraum fehlte die vordere Sitzreihe.

Als Oppositivität (,Kontrast ${ }^{\star}$, ,Vergleich) lässt sich auch der ursprüngliche semantische Beitrag des indogermanischen -(t)ero-Suffixes beschreiben, auf das das oben genannte -er-Suffix zurückgeht (vgl. Kühner/Holzweissig 1994, S. 571 f.; Streitberg 1915, S. 196; Schwyzer 1959, S. 189): ${ }^{17}$

17 Wir danken Peter Gallmann für diesen Hinweis. 
-ero-, -tero- rührten von Adverbien räumlicher Bedeutung her, wobei zunächst nur der Kontrastbegriff in Vergleichung stand: * $u p e r o-s$, oben und nicht unten befindlich', *ndhero-s, unten und nicht oben befindlich', *entero-s, innen und nicht außen befindlich', *ek(s)tero-s, außen und nicht innen befindlich', gr. $\delta \varepsilon \xi ı \tau \varepsilon \varrho o ́ s ~, d e x t e r^{6}$,

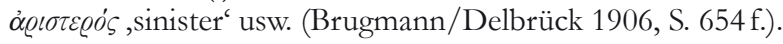

$\mathrm{Zu}$ beachten ist jedoch, dass dieser Bedeutungsbeitrag in den Vergleichssprachen bei allen dimensionalen Lokaladjektiven, unabhängig von der jeweiligen morphologischen Kodierung, zu finden ist.

Der entscheidende semantische Unterschied zwischen den genannten Lokaladjektiven und ihren adverbialen Gegenstücken besteht nun darin, dass letztere nicht oppositiv sind; ihre adnominale Verwendung induziert keine Existenzpräsupposition der oben genannten Art. Somit bedeuten die Ausdrücke in der linken Spalte von (48) nicht dasselbe wie die in der rechten (vgl. dagegen Trost 2010, S. 323).
a. die obere Schublade - die Schublade oben
b. die untere Schublade - die Schublade unten
c. die linke Tür - die Tür links
d. die rechte Tür - die Tür rechts
e. die vordere Sitzreihe - die Sitzreihe vorn
f. die hintere Sitzreihe - die Sitzreihe hinten

Ein Testfall für das Vorliegen einer oppositiven lokalen Bedeutung eines Ausdrucks $\alpha$ ist dessen Verwendbarkeit als adnominaler Modifikator eines nominalen Kopfes $\beta$ in Situationen, in denen nur ein einziger mit $\beta$ zu bezeichnender Gegenstand vorhanden ist, also bezogen auf die Beispiele in (48), in denen es nur eine einzige Schublade, Tür oder Sitzreihe zu lokalisieren gibt. In solchen Situationen ist die Verwendung eines oppositiven Ausdruck zum Zwecke der Lokalisierung inakzeptabel, da die oben genannte Präsupposition nicht erfüllbar ist. Will man sich z.B. auf die einzige Schublade eines Möbelstücks beziehen und diese gleichzeitig mit Blick auf die Dimensionen des Möbelstücks lokalisieren, so sind nur Aussagen wie (49a), nicht aber wie (49b) möglich: ${ }^{18}$

a. Die Schublade oben/unten/links/rechts/vorn/hinten klemmt.

b. Die obere/untere/linke/rechte/vordere/hintere Schublade klemmt.

Im Englischen und Polnischen sind die lokalen Dimensionsadjektive ebenfalls oppositiv, im Gegensatz zu ihren adverbialen Gegenstücken. Für das

18 Damit soll nicht gesagt werden, dass sich solche Beschreibungen in Textkorpora oder im Internet nicht finden lassen. Eine Beschreibung wie Der Nachttisch hat eine obere Schublade klingt sicher weniger umständlich als Der Nacbttisch hat im oberen Teil eine Schublade, aber sie ist dennoch nicht sprachsystemkonform, wenn der Nachttisch keine untere Schublade hat. 
Englische sind dies die Adjektive top/bottom, upstairs/downstairs, left/right, front/ back (vgl. (50)), für das Polnische die Adjektive górny/dolny, lewy/prany, przedniltylny (vgl. (51)).

ENG a. top/bottom drawer vs.

b. left/right door vs.

drawer at the top/at the bottom

c. front/back bench vs. bench at the front/at the back

d. upstairs/downstairs vs. neighbour upstairs/downneighbour stairs

(51) POL a. górna/dolna szuflada vs. szuflada na górze/na dole ,obere/untere Schublade $\quad$,Schublade oben/unten“

b. lewe/prawe drzwi vs. drzwi na lewo/na prawo ,linke/rechte Tür $\quad$,Tür links/rechts'

c. przedni/tylny rząd vs. rząd z przodu/z tyłu ,vordere/hintere Sitzreihe ,Sitzreihe vorn/hinten“

Im Französischen füllen die aus dem Lateinischen entlehnten Adjektive supérieur/inférieur, antérieur/postérieur die Lücke der sonst fehlenden adjektivischen Entsprechungen. Auch diese Adjektive sind oppositiv (vgl. (52)). Antérieur/postérieur sind mit lokaler Bedeutung fachsprachlich und finden sich in der Regel in lexikalisierten Adj-N-Verbindungen; daneben haben sie eine temporale Bedeutung, die in diesem Zusammenhang keine Rolle spielt.

FRA a. $\begin{array}{ll}\text { le tiroir supérieur/ vs. } \\ \text { inférieur } \\ \text { die obere/untere tiroir en haut/en bas }\end{array}$
$\begin{aligned} & \text { Schublade } \\ & \text { les membres antérieurs/postérieurs } \\ & \text {,die vorderen/hinteren Gliedmaßen }\end{aligned}$

Die französischen Adjektive gauche/droit sind zwar ebenfalls oppositiv und werden zur Lokalisierung von Gegenständen bei Annahme einer intrinsischen Perspektive angenommen. Sie werden daher unter anderem im Zusammenhang mit Körperteilen verwendet; vgl. l'œil gauche/droit, l'oreille gauche/droite etc. Ist eine intrinsische Perspektive nicht vorhanden oder nicht intendiert, wird ein formaler Verknüpfer - die Präposition de - gewählt, vgl. la porte de gauche/de droite (,die linke/rechte Tür). Die Attribuierung mithilfe eines formalen Verknüpfers besteht auch für andere dimensionale Lokaladverbien, vgl. Le tiroir en haut/en bas vs. le tiroir du haut/du bas; les sièges devant/ derrière vs. les sièges de devant/de derrière. Anders als die genannten Adverbien 
des Französischen sind diese de-Phrasen durchgehend oppositiv; sie können in Kontexten, in denen nur ein Gegenstand der betreffenden Art vorhanden ist (siehe oben), nicht verwendet werden:

FRA a. Prenez la porte à gauche/*de gauche.
b. le tiroir en haut/*du haut
c. les sièges devant $/ *$ de devant

Was das Ungarische betrifft, so sind die mit dem Suffix -i gebildeten Adjektive nicht-oppositiv und verhalten sich damit wie (juxtaponierte) Adverbien in den anderen Vergleichssprachen. Um oppositive Lokaladjektive zu bilden, muss auf das Suffix (-só/-số) zurückgegriffen werden. (54) zeigt eine Gegenüberstellung von nicht-oppositiven und oppositiven Attributen im Ungarischen.

$\begin{array}{cclll}\text { UNG } & \text { a. } & \text { a fenti fiók } & \text { vs. } & \text { a felsó fiók } \\ & \begin{array}{l}\text {,die Schublade oben“ } \\ \text { a lenti kép } \\ \text {,das Bild unten }\end{array} & \text { vs. } & \begin{array}{l}\text {,die obere Schublade } \\ \text { az alsó kép } \\ \text {,das untere Bild“ }\end{array}\end{array}$

Eine Antwort auf die Frage, warum dimensionale Lokaladjektive oppositiv sind, lässt sich unter Rekurs auf die Struktur (Asymmetrie) der Dimensionen und dem Konzept der Teil-Ganzes-Beziehung finden. Unter Voraussetzung einer bestimmten, fest gewählten extrinsischen Perspektive können für jeden Körper bestimmte Teile entlang der drei Dimensionen unterschieden werden. Jeder Körper hat einen Teil, der links ist, und einen Teil, der rechts ist, einen Teil, der vorne ist, und einen Teil, der hinten ist, einen Teil, der oben ist, und einen Teil, der unten ist. Aufgrund der Struktur der Dimensionen lässt sich aus dem Vorhandensein eines Teils, der links ist, auf einen Teil, der rechts ist, schließen, von einem Teil, der oben ist, auf einen Teil, der unten ist etc. Die lokale Situierung der Teile eines Körpers relativ zum gesamten Körper ist konstant; die dimensionalen Eigenschaften sind persistent, sofern man eine feste Perspektive voraussetzt. Damit können die dimensionalen Eigenschaften durch Adjektive versprachlicht werden (Teil links $\rightarrow$ linker Teil). Diesem Modell der dimensionalen Unterscheidung von Teilen folgt die dimensionale Unterscheidung kategorisierter Teile. In Analogie zu Folgerungen des Typs ,linker Teil $\rightarrow$ rechter Teil“ erhält man damit Folgerungen des Typs „linke Schublade $\rightarrow$ rechte Schublade“.

\section{Zusammenfassung}

Fassen wir abschließend die Ergebnisse für die betrachteten Sprachen zusammen: Im Ungarischen steht als Anbindungsstrategie nur die Adjektivierung zur Verfügung; Juxtaposition ist, wie wir gesehen haben, nur in spe- 
ziellen syntaktischen Strukturen möglich. Die Adjektivierung geschieht hier einheitlich mithilfe des $i$-Suffixes; die so gebildeten temporalen und topologischen Adjektive zeigen keine speziellen semantischen Effekte wie Persistenz oder Oppositivität; oppositivitätsmarkierende Adjektive können aber mithilfe eines anderen Suffixes gebildet werden (-só/-só). Mit Blick auf die anderen Vergleichssprachen verhalten sich die $i$-Adjektive des Ungarischen eher wie Adverbien. Polnisch und Französisch verfügen jeweils über zwei Anbindungsstrategien, Juxtaposition sowie Adjektivierung bzw. formale Verknüpfung. Im Polnischen sind die fraglichen Adjektive auf persistente Eigenschaften festgelegt, die dimensionalen markieren zudem Oppositivität. Analoges gilt für das Französische; auch hier werden keine lokalen de-Attribute zur Bestimmung einer zufälligen, nicht-persistenten Lokalität verwendet. Deutsch und Englisch schließlich verfügen über alle drei Strategien. Dabei weist das Englische in Bezug auf das von uns betrachtete Bedeutungsspektrum keine temporalen Adjektive auf - abgesehen von now und then, die als Adjektive aber nur beschränkt eingesetzt werden können. Dafür können die Temporaladverbien today, yesterday, tomorrow mithilfe eines Klitikons formal verknüpft werden. Die semantischen Effekte Persistenz und Oppositivität sind wie im Polnischen und Französischen auf die Anbindungsstrategien verteilt. Unterschiede zum Französischen zeigen sich im Anwendungsspektrum der formal verknüpften Attribute, das im Französischen weiter als im Deutschen ist.

Abgesehen vom Ungarischen zeichnet sich in Bezug auf die Vergleichssprachen übereinzelsprach eine Form-Funktions-Korrelation ab: Adjektivische und formal verknüpfte Attribute sind eher auf persistente Bedeutungen festgelegt, das gilt zumindest für die lokalen Attribute. Die topologischen adjektivischen und formal verknüpften Attribute sind darüber hinaus durchgehend semantisch oppositiv. Juxtaponierte Attribute drücken weder Persistenz noch Oppositivität aus, sie sind mit Blick auf diese Eigenschaften neutral.

Dass Adjektive persistente Eigenschaften kodieren, steht im Einklang mit generellen, übereinzelsprachlich dokumentierbaren Charakteristika der Wortart Adjektiv (vgl. Croft 1991, S. 65). Bei den präpositionalen Verknüpfern lässt sich annehmen, dass die persistente Bedeutungskomponente auf die ablativische Grundbedeutung der Präpositionen zurückgeht. Eine ablativische Bedeutung etabliert eine Assoziation mit einem Ursprungsort, eine (nur) persistente Bedeutung eine Assoziation mit einem Ort.

Für den Teilbereich der dimensionalen Lokalattribute ist Oppositivität der Witz der Unterscheidung zwischen adverbialen und adjektivischen bzw. formal verknüpften Attributen. Nicht-oppositive lokale Dimensionsadjektive gibt es in den Vergleichssprachen mit Ausnahme des Ungarischen nicht. Im Deutschen findet sich zwar obig, das nicht-oppositiv ist, dieses ist jedoch auf die Textdeixis beschränkt. 
Umgekehrt besteht die Funktionalität der Juxtapositionsstrategie offensichtlich darin, für die temporale oder lokale Situierung nominaler Köpfe Informationen beizusteuern, die sonst nur durch komplexere sententiale Konstruktionen (Relativsätze oder Partizipialphrasen), also der eigentlichen Domäne von Adverbien, versprachlicht werden könnten. Als juxtaponierte Attribute sind die Adverbien aber z.T. noch für temporale und lokale Information außerhalb der NP sensitiv und unterliegen daher entsprechenden Kompatibilitätsbeschränkungen. Sie verhalten sich in dieser Hinsicht so, wie sie sich auch als verb- oder satzbezogene Adverbien verhalten würden.

\section{Korpora}

BNC $=$ British National Corpus. Internet: http://corpus.byu.edu/bnc/ (Stand: 05.12.2011).

DeReKo $=$ Deutsches Referenzkorpus.

Internet: https://cosmas2.ids-mannheim.de/cosmas2-web/ (Stand: 05.12.2011).

FRANTEXT = Internet: http://www.frantext.fr (Stand: 12.12.2011).

\section{Literatur}

Anderson, Mona (1983): Prenominal genitive NPs. In: Linguistic Review 3, 1, S. 1-24.

Bauer, Laurie (1983): English word-formation. Cambridge u.a.

Behaghel, Otto (1989): Deutsche Syntax. Eine geschichtliche Darstellung. Bd. II: Die Wortklassen und Wortformen. B. Adverbium. C. Verbum. 2., unveränd. Aufl. Heidelberg.

Biber, Douglas et al. (1999): Longman grammar of spoken and written English. Harlow.

Blatz, Friedrich (1896): Neuhochdeutsche Grammatik mit Berücksichtigung der historischen Entwicklung der deutschen Sprache. 2. Bd.: Satzlehre (Syntax). 3., völlig neubearb. Aufl. Karlsruhe.

Bolinger, Dwight (1967): Adjectives in English: attribution and predication. In: Lingua 18, S. 1-34.

Brugmann, Karl/Delbrück, Berthold (1906): Grundriß der vergleichenden Grammatik der indogermanischen Sprachen. Bd. 2. Teil 1. Straßburg.

Cabredo Hofherr, Patricia (i.d.Bd.): Verschmelzungsformen von Präposition und Artikel. Deutsch und Französisch kontrastiv.

Collins COBUILD (2006): Collins COBUILD advanced learner's dictionary. 5. Aufl. Glasgow.

Croft, William (1991): Syntactic categories and grammatical relations: the cognitive organization of information. Chicago.

Curme, George O. (1922): A grammar of the German language. Designed for a thoro and practical study of the language as spoken and written to-day. Überarb. Aufl. London. 
Duden-Grammatik (2009): Der Duden. Bd. 4: Die Grammatik : unentbehrlich für richtiges Deutsch. Hrsg. v. d. Dudenredaktion. 8., überarb. Aufl. Mannheim u.a.

DWB $=$ Das Deutsche Wörterbuch von Jacob und Wilhelm Grimm auf CD-ROM und im Internet. Internet: www.dwb.uni-trier.de (Stand: 05.12.2011).

Eisenberg, Peter (2002): Morphologie und Distribution - Zur Morphosyntax von Adjektiv und Adverb im Deutschen. In: Schmöe, Friederike (Hg.): Das Adverb - Zentrum und Peripherie einer Wortklasse. Wien, S. 61-76.

Eisenberg, Peter (2004): Grundriß der deutschen Grammatik. Bd. 2: Der Satz. 2., überarb. u. aktual. Aufl. Stuttgart/Weimar.

Fleischer, Wolfgang/Barz, Irmhild (1992): Wortbildung der deutschen Gegenwartssprache. Unter Mitarbeit von Marianne Schröder. Tübingen.

Grevisse, Maurice (1993): Le bon usage. 13. überarb. Aufl. Neu hrsg. v. André Goosse. Paris/Louvain-la-Neuve.

Huddleston, Rodney D./Pullum, Geoffrey K. (2002): The Cambridge grammar of the English language. Cambridge/New York.

Klein, Wolfgang (2001): Deiktische Orientierung. In: Haspelmath, Martin et al. (Hg.): Language typology and language universals. An International Handbook. Bd. 1. (= Handbücher zur Sprach- und Kommunikationswissenschaft 20.1). Berlin/New York, S. 575-589.

Klinge, Alex (2005): The origin of Weapons of Mass Destruction. Investigating traces of lexical formation patterns in the (linguistic) history of Europe. In: Nølke, Henning et al. (Hg.): Grammatica. Festschrift in honour of Michael Herslund. Frankfurt a.M. u.a., S. 233-247.

Kühner, Raphael/Holzweissig, Friedrich (1994): Ausführliche Grammatik der lateinischen Sprache. Erster Teil. Elementar-, Formen- und Wortlehre. Darmstadt. [Unverändertert Nachdruck der 2. Auflage, Hannover 1912].

Laczkó, Tibor (2003): On oblique arguments and adjuncts of Hungarian event nominals. In: Butt, Miriam/Holloway King, Tracy (Hg.): Nominals. Inside and out. Stanford, CA, S. 201-234.

Lyons, John (1977): Semantics. Bd. 2. Cambridge u.a.

OED (2011): Oxford English Dictionary. Online-Ausgabe. Internet: www.oed.com (Stand: 05.12.2011).

Payne, John/Huddleston, Rodney/Pullum, Geoffrey K. (2010): The distribution and category status of adjectives and adverbs. In: Word Structure 3, S. 31-81.

Quirk, Randolph et al. (1985): A comprehensive grammar of the English language. London/New York.

Ramat, Paolo/Ricca, Davide (1998): Sentence adverbs in the languages of Europe. In: van der Auwera, Johan (Hg.): Adverbial constructions in the languages of Europe. (= Empirical Approaches to Language Typology 20-3). Berlin/New York, S. 187 275.

Riegel, Martin, Pellat/Jean-Christophe/Rioul, René (1996): Grammaire méthodique du français. 2. korr. Aufl. Paris. 
Schwyzer, Eduard (1959): Griechische Grammatik auf der Grundlage von Karl Brugmanns griechischer Grammatik. Syntax und syntaktische Stilistik. Vervollst. u. hrsg. v. Albert Debrunner. 2., unveränd. Aufl. (= Handbuch der Altertumswissenschaft; Abt. 2, Teil 1, Bd. 2). München.

Streitberg, Wilhelm (1915): Die Bedeutung des Suffixes -ter-. In: Indogermanische Forschungen 35, S. 196-197.

Trost, Igor (2010): Die semantische und die grammatische Sekretion am Beispiel der Komparativpositive. In: Harnisch, Rüdiger (Hg.): Prozesse sprachlicher Verstärkung: Typen formaler Resegmentierung und semantischer Remotivierung. (= Linguistik - Impulse \& Tendenzen 37). Berlin/New York, S. 317-340.

Ulvestad, Bjarne (1972): Zum postpositiven Attribut im Deutschen: Ein Becher aus Gold ... Der Mann gestern ... In: Linguistische Studien II. (= Sprache der Gegenwart 22). Düsseldorf, S. 165-180.

Wandruszka, Ulrich (1972): Französische Nominalsyntagmen. Relationsadjektivkonstruktion, ,Subst. + Subst.'-Kompositum, Präpositionalsyntagma. (= Romanica monacensia 7). München.

Wilmanns, Wilhelm (1899): Deutsche Grammatik: gotisch, alt-, mittel- und neuhochdeutsch. 2. Wortbildung. 2. Aufl. Straßburg.

Zifonun, Gisela/Hoffmann, Ludger/Strecker, Bruno (1997): Grammatik der deutschen Sprache. Bd. 1. (= Schriften des Instituts für Deutsche Sprache 7.1). Berlin/New York. 